\title{
Vorwort zur ersten Auflage
}

Das vorliegende Lehrbuch über mechanische Weberei ist für den Unterricht an Textilschulen und zum Selbstunterricht für Anfänger, Textiltechniker und Meịster bestimmt. Es soll einem Bedürfnis nach einem Unterrichtsbuche abhelfen, das auch die neuesten Fortschritte auf dem Gebiete der Weberei berücksichtigt. Bei der Bearbeitung habe ich mich von meinen langjährigen Erfahrungen im Unterricht über mechanische Webstühle leiten lassen. Überall dort, wo es zweckmäßig ist, habe ich an Stelle der Schnittzeichnungen und Skizzen perspektivische Abbildungen gesetzt, weil dadurch das Verständnis und Interesse gefördert wird. Das Ganze ist so gehalten, da $B$ auch dem Anfänger das Studium erleichtert und er systematisch in den Bau und die Arbeitsvorgänge der mechanischen Webstühle eingeführt wird. An die allgemeine Ưbersicht über die Arbeitsorgane schließen sich deshalb die charakteristischen Typen, die Bauarten der verschiedenen Webstühle an, ohne zu früh in die dem Anfänger noch unverständlichen Details einzugehen, wobei die Ausgangspunkte aller Bewegungen für die später zu besprechenden Arbeitsleistungen des mechanischen Webstuhles übersichtlich behandelt sind. Naturgemä $\beta$ muß hierauf der Antrieb und die Berechnung der Tourenzahl an Hand der einfachen Buchstabenrechnung besprochen werden, so da $\beta$ dadurch zugleich eine Einleitung für die später folgenden Berechnungen gegeben ist. Bei dem außerordentlich reichhaltigen Material, das'zur Bearbeitung vorlag, habe ich eine sorgfältige Auswahl getroffen und nur das unbedingt Notwendige und das, was sich in der Praxis bewährt hat, besprochen. Uberall ist auf die Einordnung der einzelnen Mechanismen in das Ganze, d. h. auf ihre Verbindung mit andern Teilen des Webstuhles und ihre Zugehörigkeit zu den verschiedenen Webstuhlarten hingewiesen worden.

Aachen, im April IgIr.

H. Repenning. 